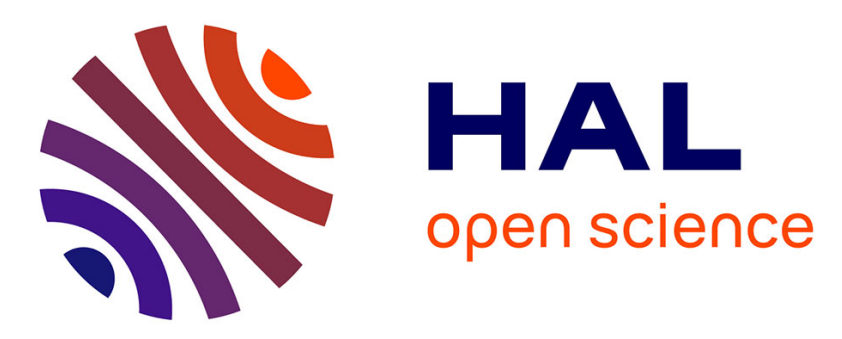

\title{
The role of Monte Carlo simulation in understanding the performance of proton computed tomography
}

George Dedes, Jannis Dickmann, Valentina Giacometti, Simon Rit, Nils Krah,

Sebastian Meyer, Vladimir Bashkirov, Reinhard Schulte, Robert Johnson, Katia Parodi, et al.

\section{To cite this version:}

George Dedes, Jannis Dickmann, Valentina Giacometti, Simon Rit, Nils Krah, et al.. The role of Monte Carlo simulation in understanding the performance of proton computed tomography. Zeitschrift fur Medizinische Physik, 2022, 32, pp.23-38. 10.1016/j.zemedi.2020.06.006 . hal-02920133

\section{HAL Id: hal-02920133 \\ https://hal.science/hal-02920133}

Submitted on 2 Oct 2020

HAL is a multi-disciplinary open access archive for the deposit and dissemination of scientific research documents, whether they are published or not. The documents may come from teaching and research institutions in France or abroad, or from public or private research centers.
L'archive ouverte pluridisciplinaire HAL, est destinée au dépôt et à la diffusion de documents scientifiques de niveau recherche, publiés ou non, émanant des établissements d'enseignement et de recherche français ou étrangers, des laboratoires publics ou privés. 


\title{
The role of Monte Carlo simulation in understanding the performance of proton computed tomography
}

\author{
George Dedes \\ Department of Medical Physics, Fakultät für Physik, Ludwig-Maximilians-Universität \\ München (LMU Munich), Garching, Germany \\ Jannis Dickmann \\ Department of Medical Physics, Fakultät für Physik, Ludwig-Maximilians-Universität \\ München (LMU Munich), Garching, Germany \\ Valentina Giacometti \\ The Patrick G Johnston Centre for Cancer Research, Queen's University of Belfast, \\ Northern Ireland Cancer Centre, Belfast, Northern Ireland, United Kingdom \\ Simon Rit \\ University of Lyon, CREATIS, CNRS UMR5220; Inserm U1044, INSA-Lyon, Université \\ Lyon 1, Centre Léon Bérard, Lyon, France \\ Nils Krah \\ University of Lyon, CREATIS, CNRS UMR5220; Inserm U1044, INSA-Lyon, Université \\ Lyon 1, Centre Léon Bérard, Lyon, France \\ University of Lyon, Institute of Nuclear Physics Lyon (IPNL), CNRS UMR 5822, \\ Villeurbanne, France \\ Sebastian Meyer \\ Department of Medical Physics, Fakultät für Physik, Ludwig-Maximilians-Universität \\ München (LMU Munich), Garching, Germany \\ Vladimir Bashkirov \\ Division of Biomedical Engineering Sciences, Loma Linda University, Loma Linda, CA, \\ United States of America \\ Reinhard Schulte \\ Division of Biomedical Engineering Sciences, Loma Linda University, Loma Linda, CA, \\ United States of America \\ Robert Johnson \\ Department of Physics, U. C. Santa Cruz, Santa Cruz, CA, United States of America \\ Katia Parodi
}




\author{
Department of Medical Physics, Fakultät für Physik, Ludwig-Maximilians-Universität \\ München (LMU Munich), Garching, Germany \\ Guillaume Landry \\ Department of Radiation Oncology, University Hospital, LMU Munich, Munich, Germany \\ German Cancer Consortium (DKTK), Munich, Germany \\ Department of Medical Physics, Fakultät für Physik, Ludwig-Maximilians-Universität \\ München (LMU Munich), Garching, Germany
}

\begin{abstract}
Proton computed tomography ( $\mathrm{pCT}$ ) is a promising tomographic imaging modality allowing direct reconstruction of proton relative stopping power (RSP) required for proton therapy dose calculation. In this review article, we aim at highlighting the role of Monte Carlo (MC) simulation in pCT studies. After describing the requirements for performing proton computed tomography and the various $\mathrm{pCT}$ scanners actively used in recent research projects, we present an overview of available MC simulation platforms. The use of MC simulations in the scope of investigations of image reconstruction, and for the evaluation of optimal RSP accuracy, precision and spatial resolution omitting detector effects is then described. In the final sections of the review article, we present specific applications of realistic Monte Carlo simulations of an existing pCT scanner prototype, which we describe in detail.
\end{abstract}

Keywords: proton computed tomography, Monte Carlo simulation, proton therapy, relative proton stopping power

\title{
1. Introduction
}

X-ray computed tomography relies on statistical attenuation measurements as a means to obtain line integrals through the linear attenuation coefficient of an object, allowing three-dimensional (3D) reconstruction of that quantity.

${ }_{5}$ Cormack pointed out in the early 1960s that we are not restricted to x-rays to obtain line integrals; charged particles, such as protons, which lose energy continuously, may serve the same purpose [1, 2. While in those initial papers Cormack expressed doubts regarding the feasibility of using protons, Koehler subsequently showed that proton radiographs could be obtained with a high 10 contrast 3. Cormack and Koehler presented preliminary experimental results supporting the use of protons for tomography [4, followed by work from Hanson and colleagues [5, 6, 7, 8, These seminal publications report that protons have a dose advantage over $\mathrm{x}$-rays at equivalent noise, and that spatial resolution is 
poorer; Schulte 9], Poludniowski [10] and Johnson [11] provide good historical overviews ranging from early 1960s to 2018.

As opposed to x-rays, protons theoretically allow the acquisition of line integrals on a proton-per-proton basis, as long as they have sufficient initial energy to cross the object [12]. The relative proton stopping power between human tissues and water, which is generally the quantity reconstructed in proton to20 mography, is essentially energy independent; thus measuring the proton kinetic energy difference before and after the object allows the determination of the water equivalent path length (WEPL). In other words, in proton CT, we perform line integrals through the energy-independent relative stopping power (RSP) and can reconstruct this quantity, which is needed for proton therapy treatment 25 planning.

While x-rays interact in a sparse fashion, protons undergo myriad deflections along their path due to multiple Coulomb scattering (MCS), and assuming straight ray-lines between source and detector will lead to images with suboptimal spatial resolution 13. Measuring the location and direction of travel

30 of each proton before and after the object allows the reconstruction of a curved path which approximates the true proton path. It is along these curved paths that we now assume the RSP line integrals are performed [14. Thus, a proton CT scanner equipped with proton trackers and an energy detector allows use of proton-per-proton information in image reconstruction, something which we call

35 list-mode in analogy to the detection of individual lines of response in positron emission tomography imaging. Specialized algorithms have been developed to account for the curved paths, either using iterative reconstruction [15, 16, 17] or variations of filtered-backprojection [18, 19, which allow the creation of volumetric RSP images. Alternatively to that, integration-mode scanners combine the information of several protons to estimate their mean energy loss.

Designing a scanner capable of optimally obtaining proton-per-proton measurements for image reconstruction requires balancing several factors which contribute to the scanner's performance. Spatial resolution is affected by MCS in the object and the detector, by the proton trackers' sampling resolution and 45 their distance to the object, as well as by the accuracy of the proton curved path estimation. RSP noise will depend on the energy resolution of the detector, as well as on the energy straggling experienced by each proton in the object and in the detector. The dimensions of the energy detector and trackers will naturally set the field of view (FOV) of the scanner, but will also impact the 50 count rate at which the scanner can be operated, which further depends on the design of each component individually. Operating a list-mode or integrationmode scanner, with or without tracking will lead to differences in performance. For some of the aspects above, Monte Carlo (MC) simulations offer an effective way of obtaining insight on the impact of design choices on scanner performance.

${ }_{55}$ Furthermore, given the scarcity of functioning pCT scanner prototypes, several authors have turned to simulation to answer research questions. In this article, we will review the use of MC simulation in proton $\mathrm{CT}$, which includes understanding theoretical limits, comparing scanner designs as well as furthering our understanding of the behavior of a specific pCT scanner. The ability to per- 
form Monte Carlo simulation allowing a full understanding of the imaging chain requires knowledge of minute details of that chain. To be able to fully exploit such simulations, it is desirable that they yield output capable of being fed to existing data processing and reconstruction software. Thus, detailed knowledge of the data formation process of the scanner is required to set up the simula-

65 tion correctly. With this review, we opted to dissect these details, using the simulation of the phase II pCT scanner [20] as an example.

\section{2. pCT scanner requirements}

In the following, the general requirements of a list-mode pCT scanner are summarized, separately focusing on characteristics of tracking and energy de-

70 tectors. We also briefly describe how different pCT scanner prototypes address these requirements.

\subsection{Tracking requirements}

Tracking detectors are used in pCT to measure the proton trajectory before its entry into the object and after its exit. Ideally, both the proton position 75 and direction should be measured at entry and exit, but if the incoming beam direction is already known, then a position measurement may be all that is needed at the entry.

Typically, the tracking instrument measures the locations where the proton passes through planes of thin tracking detectors. Each plane may measure both so transverse coordinates simultaneously, as in the case of pixelated detectors, but simpler (and often more practical) detector planes may measure only a single coordinate. A distinct disadvantage of the latter configuration is that, in the case that more than one proton passes through the instrument at a time, as is likely to happen frequently if high rates are desired, then additional detector planes 85 oriented at different "stereo" angles may be needed in order to associate properly the individual measurements into tracks. Even such stereo layers will not be sufficient for disentangling the hits from multiple simultaneous protons if the protons are confined within a narrow pencil beam (causing higher count rates for the single coordinate measurements). The alternative is to work with detectors

90 that measure just a single coordinate while trying to make the electronics as fast as possible. For example, the Phase-II pCT scanner discussed later, based on silicon-strip detectors, uses a tracker timing window of about $150 \mathrm{~ns}$ and works effectively at proton rates of up to $1.2 \mathrm{MHz}$ with a broad beam without acquiring excessive numbers of two-proton events 20. Significantly faster electronics, consuming more power, and possibly shorter strips would be required to decrease the scan time from 5 minutes to less than a minute by increasing count rates.

The tracking spatial resolution requirements are relaxed compared to what is technically possible, because MCS in the phantom itself limits the image resolution. For the same reason, there is no advantage in making the detectors 100 themselves as thin as possible in terms of scattering material. For example, for a water phantom of $20 \mathrm{~cm}$ thickness, the uncertainty in the proton path due to 
MCS in the phantom is about $0.5 \mathrm{~mm}$ near the center of the phantom [14, so it would be pointless to try to strive for point resolutions in the detector planes better than the order of $0.1 \mathrm{~mm}$.

High detection efficiency in individual tracking planes, with low noise, is essential for pCT unless a lot of redundant planes are added. Loss of a single hit seriously compromises the measurement of a proton track, and any loss of events to inefficiency increases the dose to the patient and the duration of the scan.

${ }_{110}$ A more detailed discussion of tracking requirement for a pCT scanner can be found in Section 3 of Ref. [11.

\subsection{Energy detector requirements}

The current estimates of range uncertainties in proton therapy [21] and published results of a multicenter audit of quality assurance data 22] imply that 115 RSP accuracy and resolution better than $1 \%$ are desirable for pCT images used for treatment planning. The same requirements are usually assumed for the RSP line integral, the WEPL.

Another key requirement is the ability of a pCT scanner to acquire data suitable for tomographic reconstruction within a clinically acceptable time span. As mentioned in [10, 23], approximately $10^{8}$ protons are needed in order to achieve the acquisition of a pCT scan with reasonable imaging noise. This translates to data acquisition rates of $1 \mathrm{MHz}$ to $10 \mathrm{MHz}$, for scan times ranging from a few minutes to about $10 \mathrm{sec}$. At the same time, the detector size and aperture should minimally accommodate the scan of an adult head (approximately

$12530 \mathrm{~cm}$ ). Furthermore, the WEPL range detectable by such a system should span from 0 up to about $35 \mathrm{~cm}$ for a body scan, which also defines the minimum longitudinal size of a WEPL detector, since all protons must stop in the detector. Alternatively, multiple sequential energy scans can be used as means to cover the desired dynamic WEPL range with a more compact system [24.

The two main WEPL detector design concepts are that of the calorimeter and of the range telescope. A calorimeter consists of a non-segmented detector in the longitudinal direction, which aims at determining the traversed WEPL in the object by measuring the residual energy of protons. A range telescope on the other hand, comprises a large number of thin detector elements (stages)

135 with absorbers in between and the WEPL determination comes from identifying the stage in which an impinging proton stops.

There are few factors that can affect the WEPL measurement resolution. Firstly, the proton beam energy spread, assuming that no upstream energy measurement is performed. Secondly, the range/energy straggling of protons in the object scanned. Finally, the energy resolution of the detection system and the range/energy straggling in the WEPL detector system itself, assuming negligible contribution from the tracker. The goal of a WEPL detector is to approach the inherent WEPL resolution limit set by range straggling. The latter is approximately $1.1 \%$ of the total proton range in water [25], thus approximately 
For a calorimeter, the WEPL resolution is a function of the proton range straggling in the object and the energy resolution of the calorimeter [23. As shown in previous MC studies [10, 23], in order for a calorimeter to reach the theoretical limit of WEPL resolution for the maximum WEPL of a $200 \mathrm{MeV}$ proton beam, an energy resolution of $1 \%$ or better is required.

In the case of a range telescope, the WEPL resolution is a function of the total proton range straggling (in the object plus in the range telescope) and the uncertainty of the stopping position in the range telescope, which is proportional to the stage thickness. As elaborated in [10, 23, the thickness of each stage 155 should not exceed $1.5 \%$ of the total proton range, resulting in the requirement for 60 to 100 stages.

Bashkirov et al. 23 explored the concept of a hybrid WEPL detector, namely a multistage calorimeter detector with few stages (less than 10). According to their investigations, the WEPL resolution for such a design is a function of the total proton range straggling up to the stage in which the proton stopped (similarly to a range telescope) and the energy resolution in the stopping stage (similar to a calorimeter). Their design requires fewer read-out channels than a range telescope and is less demanding in terms of energy resolution of each single stage. They concluded that the theoretical limit of $1 \%$ WEPL resolution can be achieved with a $2 \%$ energy resolution in each stage.

\subsection{Summary of prototypes and requirements they cover}

Several efforts to build prototype systems for pCT have been active over the past decade, although the first attempts in that direction date back to around 1980. See references [11] and [10] for extensive reviews of this field. The main developments since those reviews were published are further developments of the PRaVDA system [26, based on silicon-strip detectors; an effort to build a range telescope based on monolithic active pixel sensors [27; and first protonradiography results from the ProtonVDA system [24].

The prototype scanners currently in use in research appear to be, from the re175 cent publication record, the Phase-II [20], the PRaVDA [26] and the ProtonVDA 24] pCT scanners. The Phase-II scanner satisfies several of the requirements for $\mathrm{pCT}$ and has been used extensively in research since 2014. Its aperture is wide enough to image head-sized rotating objects, but is limited in rate by its readout requirements such that 5 minutes or more are needed for a complete CT scan. Furthermore, its design was optimized for imaging with a cone beam or a large beam spot, such that its tracking efficiency decreases significantly, due to pileup, when used with pencil beams.

The PRaVDA system is based entirely on silicon-strip detectors to instrument both the tracker and a range detector. Unlike the Phase-II scanner, it 185 includes additional stereo layers in the tracker, and given that the range detector is finely instrumented by silicon strips, the system should be able to measure multiple protons simultaneously. In general it should be much faster than the Phase-II scanner, although the example CT scan reported in [26] operated at 1.4 MHz. The main limitation of this prototype is its small aperture, which has allowed it to scan only objects less than about $8 \mathrm{~cm}$ in size. 
ProtonVDA is based on scintillating fibers for tracking and a monolithic scintillator for residual-range measurement and it is designed specifically to operate with pencil-beam scanning. Its tracking detectors measure only position, not direction, before and after the object, which should limit its spatial resolution. Also, its energy detector requires modulation of the beam energy to keep the Bragg peak contained within the relatively small scintillator, matching well the capabilities of a pencil-beam scanning system. The aperture is large, at $40 \mathrm{~cm} \times 40 \mathrm{~cm}$, and well suited to imaging the human body. The system has already been demonstrated to work well for proton radiography [24, but in 200 principle could also be used for pCT if the patient is rotated, for example in a rotating chair.

\section{Monte Carlo simulation platforms}

\subsection{Simulation frameworks}

Protons traversing matter experience both electromagnetic and nuclear interactions which give rise to a variety of secondary particles. Therefore, an adequate simulation of a proton image acquisition should be performed by general purpose Monte Carlo (MC) codes.

The two main particle transport MC frameworks that have been used for proton imaging studies are the Geant 4 toolkit [28] and the FLUKA simulation package 29]. In addition, two Geant4-based simulation applications, GATE [30] and TOPAS [31, have been frequently utilized for proton imaging studies.

\subsection{Physics and models}

FLUKA and Geant4 provide detailed modelling of transport and interaction mechanisms of protons, heavier ions and their secondaries, in the energy range relevant for particle therapy. Both codes have been extensively benchmarked against experimental data and intercompared. Results of recent validation and comparison studies can be found in [32, 33, 34, 35, 36, 37, 38.

Geant4 and FLUKA rely on the Bethe formula with additional corrections for the calculation of continuous energy loss of charged particles. Based on the energy loss, the WEPL traversed in an object can be derived and eventually used to reconstruct RSP. In addition to energy loss, MCS in the object and the detectors also plays a major role in image formation, as it may increase imaging noise and deteriorate spatial resolution. Molière's and Urban's formalism are the basis of the FLUKA and Geant4 MCS models, respectively. Nuclear interactions 225 contribute to beam attenuation and creation of secondary particles. For energy loss $\mathrm{pCT}$, they result in deterioration of the $\mathrm{pCT}$ image if not removed from the set of protons used for reconstruction. Together with MCS, they have been recently utilized in attenuation and scattering pCT studies by 39, 40, 41.

Table 1 summarizes the most widely adopted physics models in published pCT studies. In FLUKA, the default setting HADROTH invokes all the models necessary for accurate simulation of proton imaging applications. Geant4 allows the user to tailor a custom set of models. Often, the QGSP_BIC_HP physics list 
has been employed and based on that, users opt for different electromagnetic physics packages depending on the requirements for accuracy and speed.

Table 1: List of models describing different types of particle interactions.

\begin{tabular}{c|c||c}
\hline Process & FLUKA & Geant4 \\
\hline & & G4EmStandardPhysics_option3/4 \\
Electromagnetic & EMF & G4EmLivermorePhysics \\
& & G4EmStandardPhysics \\
Hadron inelastic & PEANUT & G4HadronPhysicsQGSP_BIC_HP \\
Hadron elastic & parametrized & G4HadronElasticPhysicsHP \\
Nucleus inelastic & RQMD-BME & G4IonBinaryCascadePhysics \\
\hline Model set & HADROTHE & QGSP_BIC_HP \\
\hline
\end{tabular}

235 example is the case where the optical light simulation and transport is omitted and the signal from a scintillator is parameterized as a function of the deposited energy, as demonstrated by Dickmann et al. 42 and briefly described in section 6.2. In that case, benchmarking against experiments is essential. Usually the 265 readout electronics of the detector components are not simulated in particle transport codes. 


\section{Reconstruction algorithms}

MC simulations can generate input data for a reconstruction algorithm to analyze the effect on CT image quality of a given hardware design or to evaluate

270 scanners used integration-mode detectors and straight line paths were assumed for the protons. The reconstruction problem is then very similar to that of $\mathrm{x}$-ray $\mathrm{CT}$ and the same algorithms have been used, e.g., filtered backprojection (FBP) [5, 6, 7, 8, 13. The limited spatial resolutions of these early pCT images led to the curved proton path (see section 5.2). Accounting for these estimated curved paths in the reconstruction has been the subject of extensive investigations, typically based on MC simulations of list-mode pCT scanners.

Iterative techniques were first developed because a non-linear path can be Similaty accounting for the uncertainty in the path estimation [45. Similarly to reconstruction techniques using straight lines, a linear system of equations is obtained and the same reconstruction techniques can be used. Most algorithms attempt to find the solution which best fits the data in the least-squares sense but they differ in how they solve the problem [46, 47, 48, 49. They can also differ on prior assumptions, e.g., that the reconstructed object should be piecewise constant [16, 50, 51] or similar to an x-ray prior [50, 52.

Direct FBP algorithms can also use curved paths in different ways. Some authors have attempted to use the curved paths before the reconstruction to bin 290 the list-mode data in a set of two-dimensional radiographs [53, 54, 55]. Others have backprojected the curved paths in the three-dimensional object space with filtering occurring before [18, 56] or after [19] the sum over all detector positions. Mainly two studies compared a few pCT reconstruction algorithms based on Monte Carlo simulations only. Hansen et al. 49 compared three iterative reconstruction algorithms and one direct reconstruction algorithm. More recently, Khellaf et al. [57] compared five direct reconstruction algorithms. Both studies evaluated spatial resolution from the modulation transfer function measured in different phantoms. Limited differences were observed, which suggests that the spatial resolution is mainly limited by the uncertainty of the proton path estimates (for more details, see section 5.3). The same applies to RSP accuracy and resolution, which was evaluated using simulations of an electron density phantom used in clinical practice made of known plastic materials mimicking human tissues, and also only showed small variations. The main difference between these algorithms is the computational time, which is larger for iterative algorithms.

\section{MC simulations with ideal detection and analytical studies}

In the following sections we will summarize the findings of several MC simulations studies that assume no detector effects, regarding RSP accuracy and 
precision, spatial resolution, and modeling of the biological effect of the imaging

\subsection{Studies on $R S P$ accuracy and $R S P$ resolution}

Ideal studies use scoring planes to score the location where a given proton crosses the plane, as well as its direction vector and energy. Coordinates and direction vectors then serve as input for curved path estimation, and the energy difference between the planes is used to calculate the WEPL of the proton, using a pre-calculated water stopping power versus energy tabulation.

Several pCT publications have made use of ideal MC simulations, such as the work of Schulte et al. [58] who used Geant4 simulations to successfully validate a theoretical prediction of pCT noise levels at the center of a water cylinder. 320 Ideal simulations have also been used by Hansen et al. 49] to show that iterative and analytical reconstruction methods can reach similar levels of image quality with ideal inputs, down to $1 \mathrm{mSv}$ dose levels. Such simulations have also been used to provide a bound of RSP accuracy in studies by Hansen et al. 43 and Dedes et al. 59 comparing pCT accuracy to dual energy x-ray CT (DECT), 325 and by Arbor et al. 60] against single energy x-ray CT (SECT), showing a mean absolute percent error of $0.2 \%$ for pCT under ideal conditions.

Rädler et al. 61] used ideal simulations to study pCT noise in 2D and showed an increase by up to $40 \%$ towards the object's edge. Collins-Fekete et al. 62 performed a confirmation study establishing an analytical noise model based 3зо on ideal MC simulations and supporting the findings of Rädler et al. 61] and Schulte et al. [58.

It may also be desirable to ignore detector effects when evaluating the baseline performance of different ions than protons [17, 63], however as shown by Volz et al. 64, heavier ions may interact differently than protons with energy

335 detectors. This can be investigated by performing MC simulations with nuclear interactions disabled, as in for example Volz et al. 65.

In general, while ideal simulations may provide valuable insight in the image formation process, one should acknowledge that they will most likely not account for the significant impact on image quality stemming from the proton detection 340 processes. They serve as an upper limit of image quality achievable with a pCT system.

\subsection{Studies on proton path estimation}

Several mathematical formalisms to estimate the most likely path (MLP) of a proton have been proposed or studied in the literature. All follow the same concept: they apply some kind of likelihood maximisation to estimate the most likely path which a proton has taken across an object given its position and direction upstream and downstream of the object. They differ either in the way of modelling MCS or how they account for material properties. All currently available MLP estimation methods make the assumption that angular dispersion 350 due to MCS is approximately Gaussian. This is justified by the fact that events recorded by a proton $\mathrm{CT}$ scanner are post-processed to filter out protons which 
have experienced nuclear interactions in the medium. As a side-effect, this also eliminates events with large scattering angles, i.e. those which fall outside of the Gaussian distribution. Most of the MLP models also assume that the medium is homogeneous and water-equivalent.

The works by Williams [66, Schulte et al. 14] and Collins-Fekete et al. [55] relied on MCS models commonly used in particle physics [67, the latter with a refinement proposed by Gottschalk et al. 68. Li et al. [46] used Monte Carlo simulations to compare straight-line path, MLP and a cubic spline approxima360 tion, concluding that MLP and cubic splines yielded optimal resolution. Their cubic spline path was used in subsequent Monte Carlo studies of pCT [17, 50. The comprehensive study by Erdelyi 69] investigated some of the approximations made by the MCS models (small angle approximation, correction terms). The MLP estimation presented in Collins-Fekete et al. 70] used a cubic spline path with an empirical parametrisation based on Monte Carlo simulations rather than an explicit MCS model. Krah et al. 71 showed that the MLP can effectively be described as a polynomial (of at least order three) if the energy-loss of protons as a function of depth in the medium is described as a polynomial. The differences between these MLP models in terms of estimation accuracy is

370 only a few percent and probably negligible once tracker uncertainties are also considered.

Collins-Fekete et al. 72, Brooke and Penfold 73] proposed a method to account for inhomogeneous material composition along the MLP based on an a priori image. Khellaf et al. 74 investigated the impact of material inhomo375 geneities normal to the path direction. As a conclusion of these three works, approximating patient geometries by homogeneous water volumes is sufficiently accurate for MLP estimation. Finally, since most MLP formalisms are validated via $\mathrm{MC}$ simulation, it is worthwhile mentioning that there is a few percent variation when comparing MCS models implemented in Geant4 (and other MC codes) among each other and with experimental data [75.

\subsection{Studies on spatial resolution}

The spatial resolution of $\mathrm{pCT}$ is limited due to MCS. Proton trajectories are therefore intrinsically unknown and one can only estimate the most likely path based on the measured kinematic properties. The intrinsic uncertainty envelope around the MLP is approximately Gaussian because MCS is largely governed by Gaussian statistics. In practice, a small object in a phantom (say a thin rod parallel to the rotation axis) results in a smeared spot in the reconstructed CT image. The size of this spot is directly linked to the uncertainty of the most likely path estimate and depends on the location of the object because

390 MCS depends on the amount of traversed material. Ideal MC simulations of list-mode systems record proton positions with perfect accuracy and MCS is the only source of blurriness. Objects at the outer edge of a phantom appear sharp in these simulations and increasingly blurrier towards the center.

In real trackers, the precision of position measurements is limited by the 395 size of the strips, pixels, or fibers (depending on the technology) and direction measurements additionally depend on the distance between tracker pairs (the 
further apart the more precise). This adds an extrinsic uncertainty to the most likely path estimate and degrades the spatial resolution in real data compared to ideal simulations.

Alternatively, some authors have proposed (semi-)analytical methods to calculate the expected spatial resolution based on the tracker configuration and beam properties. Bopp et al. [76] used analytical methods to study the impact of tracker plane spacing, the strip pitch and the trackers' thickness in case of list-mode proton CT systems. Schneider et al. 77, 78, studied a wider range 405 of selected set-ups including those which only measure proton positions and analysed the impact of angular confusion of the beam on spatial resolution. The work of Krah et al. 79] provides a general framework to estimate the spatial resolution achievable with a proton $\mathrm{CT}$ system, including integration-mode ones. The underlying idea is the following: a list-mode scanner which does not measure directions can be modelled as a "full" scanner where the uncertainty of one tracking plane per tracker pair is numerically infinite. Similarly, a proton CT system without upstream trackers using Gaussian pencil beams is like a list-mode system with a positional uncertainty equivalent to the beam spot size. The same mathematical formulas thus cover all systems if the numerical 415 values of uncertainty parameters are appropriately chosen.

One can extract the following guidelines from the above-cited studies: "Full" systems are superior to all other variants of $\mathrm{pCT}$ scanners with a spatial resolution about 2-3 times better than in scanners which only measure the protons' positions. In both cases, the scanner should be placed as close to the imaged ${ }_{420}$ object as possible because spatial resolution degrades drastically with increasing object-to-tracker distance, e.g. from $0.8 \mathrm{lp} / \mathrm{mm}$ at $10 \mathrm{~cm}$ to $0.3 \mathrm{lp} / \mathrm{mm}$ at $40 \mathrm{~cm}$ with a list-mode scanner [79]. Integrated mode systems are viable only in combination with pencil beam scanning, not with passive scattering delivery, but provide a spatial resolution 3-10 times inferior to that of list-mode scanners [79].

\subsection{Studies on multi-modality tomographic imaging}

Proton imaging techniques making use of a limited number of projections have also been used to obtain or refine 3D RSP maps. For example, Schneider et al. [80] proposed using a single proton radiography of the water equivalent ${ }_{430}$ thickness of a patient to refine the CT-value to RSP calibration curve by minimizing the difference of a digitally reconstructed radiography and the proton radiography. More recently, Doolan et al. 81] used a flat panel imager and time varying dose patterns created by a range modulator wheel to obtain the radiographies in their CT-value to RSP optimization, a radiography and tomography

435 technique based on prior work at the same institution [82, 83, 84]. Zhang et al. [85. performed phantom experiments using the same radiography approach and concluded that a majority of pixels $(>50 \%)$ in a given radiography may need to be rejected due to range mixing, requiring at leat 12 projections to optimize $90 \%$ of voxels. Such multi-modality approaches have been studied with Monte

${ }_{440}$ Carlo simulation by Hansen et al. [50] who explored the feasibility of hybrid iterative reconstruction methods using an x-ray CT prior and a reduced angular 
range of pCT projections. Collins-Fekete et al. [86] studied the use of only one or two list mode proton radiographies for CT-value to RSP optimization with Monte Carlo simulation. Krah et al. [87] investigated, also using idealized Monte Carlo simulations, the use of radiographies acquired with pencil beam scanning and a range telescope. Naturally, the accuracy of such methods is limited by the concordance between the x-ray CT prior and the anatomy captured by the radiography. Krah and Rit [88] proposed using in-room cone beam computed tomography images as prior to address this issue in their simulation study. To 450 address the same issue, Palaniappan et al. [89] recently proposed a 2D-3D deformable image registration that used a limited number of proton radiographies in order to update the anatomy captured by the planning x-ray CT.

\subsection{Studies on biological modelling}

While being a compelling feature for therapy, the elevated linear energy transfer (LET) of proton or, especially, heavier ion beams compared to sparsely ionizing x-rays could turn into an undesired side effect for imaging applications. Since potential biological effects could ultimately constrain the achievable image quality at a given tolerable imaging dose, a careful consideration is required. For relevant ion CT scenarios, event-by-event track structure simulations [90]

460 are not feasible due to the unsustainable amount of required computational resources. Therefore, a common approach consists in using a combined multiscale model by incorporating radiobiological information from biophysical models into condensed history MC codes 91 .

Hansen et al. 17] used an idealized MC simulation of the Phase-II pCT scanner to compare the image quality of different ions at equivalent dose. Special care was taken in defining the computed tomography dose equivalent index (CTDEI), an alternative to the standard computed tomography dose index (CTDI). To do so, ICRP LET-dependent quality factors were used when scoring the dose in the simulations [92. They evaluated accuracy and resolution from images 470 reconstructed iteratively. They concluded that Helium ions gave the best spatial resolution, and that the minimal doses required to achieve sufficiently low systematic errors were $10 \mathrm{mSv}$ for carbon ions, $5 \mathrm{mSv}$ for helium ions and 1 $\mathrm{mSv}$ for protons.

In Meyer et al. 44 biological dose computations using the mechanistic repair-misrepair fixation (RMF) model 93] were performed. The RMF model has been used to investigate the radiobiological effects related to proton, helium and carbon ion CT at a physical dose of around $2 \mathrm{mGy}$ [4] using the FLUKA MC code [29, 94]. The relative biological effectiveness (RBE) for double-strand break (DSB) induction relative to a $130 \mathrm{kVp}$ x-ray spectrum with $0.8 \mathrm{~mm}$ beryllium filtration was $0.82,0.84$ and 0.95 for proton, helium and carbon ion CT, respectively. The corresponding $\mathrm{RBE}$ for reproductive cell death using a reference tissue radiosensitivy of $(\alpha / \beta)_{X}=10$ Gy was $0.82,0.85$ and 0.97. DSBs leading to chromosome aberrations are considered an important biological endpoint for imaging applications [4]. While the validity of transferring models

485 originally developed for radiation therapy into the low dose regime is questionable, there is strong evidence that DNA damage is a linear function of dose down 
to around 1 mGy. The aforementioned results indicate that ion CT could yield reduced radiobiological implications compared to diagnostic x-ray CT, since the patient is solely receiving dose from the plateau of the Bragg-curve, being a low-LET region. Furthermore, recent experimental radiobiology investigations for proton $\mathrm{CT}$ and initial results seem to confirm the simulation findings [95.

\section{Phase-II pCT scanner modelling and data processing}

In the second half of this review article, we will focus on an operational pre-clinical pCT scanner to illustrate the potential of MC simulation in $\mathrm{pCT}$.

495 We will provide details on studies modelling the Phase-II pCT scanner shown in Fig. 1, as well as on publications applying this simulation framework to research questions. While studies focusing on this scanner prototype, the longest in operation, have been selected to be discussed in detail, Monte Carlo methods were used to investigate other scanner prototypes as well [96, 97, 53, 98, 99, 100,

500 101, 102, 27. Since these studies did not explicitly investigate the influence of the scanner on image quality for human-scale objects, we have chosen not to discuss them extensively.

(a) phase II PCT prototype scanner

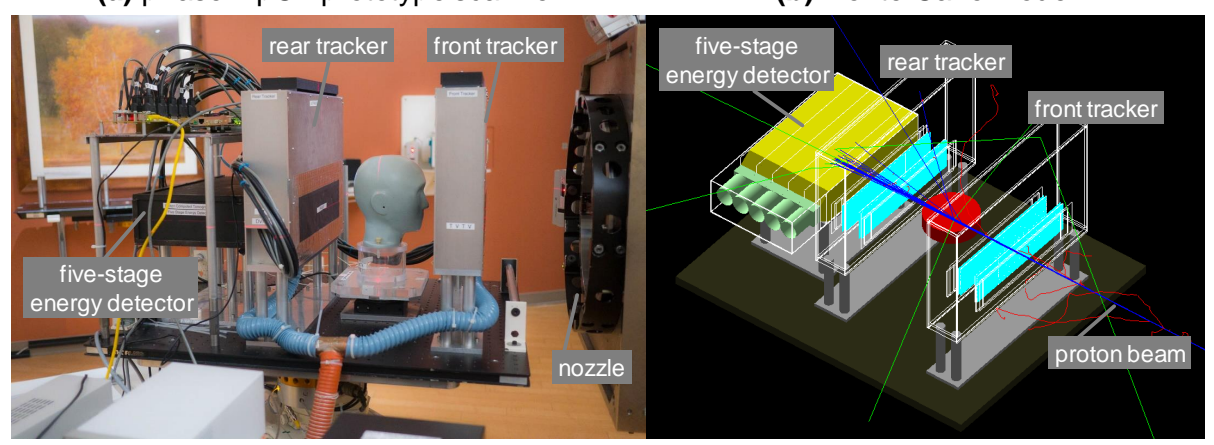

Figure 1: (a) The Phase-II pCT scanner mounted in the treatment room of the Northwestern Medicine Chicago proton scanner and (b) the corresponding three-dimensional model used for Monte Carlo simulation of pCT scanner acquisitions.

\subsection{Tracker}

The tracker of the Phase-II pCT scanner consists of two tracker modules, made of silicon strip detectors mounted on either sides of a rotation platform, equidistant from the isocenter.

The front and rear trackers consist of two paired silicon strip detector (SSD) planes with vertical and horizontal strip orientation, respectively [103. The thickness of each SSD is $0.4 \mathrm{~mm}$, and the strip pitch is $0.288 \mathrm{~mm}$. The plane with vertical strips (t-plane) is formed by 1536 strips and the plane with horizontal strips (v-plane) by 384 strips. The data are processed by 12 FPGAs (1 per 
v-plane and 2 per t-plane) mounted on the same circuit boards that carry the SSDs [104.

The tracker geometry described above was reproduced in Geant 4 v.10.1 [105], as shown in Fig 2, The SSD strips were modelled as sensitive silicon volumes; simulated strips were grouped according to strip, chip, SSD and FPGA number used by the data acquisition (DAQ) system as shown in Fig. 2. Vertical and horizontal strips are numbered from 0 to 63 . Each group of 64 strips is associated to one chip number. The chips are numbered from 0 to 11 in the t-planes (1536 520 vertical strips), and from 0 to 5 in the v-planes (364 horizontal strips). Each group of 6 chips is associated to one SSD number (SSD 0, 1, 2, and 3 in the t-planes, SSD 0, 1 in the v-planes). The SSD in each plane are processed by one FPGA per v-plane (FPGA numbered from 0 to 3 ) and two FPGA's per t-plane (FPGA numbered from 4 to 11). Therefore, when a proton hits a sensitive strip, from the coordinate of the hit, the simulation traces back the strip, chip, SSD and FPGA numbers correspondent to that event. These are converted to locations in the $\mathrm{pCT}$ scanner coordinate system during the postprocessing step described in section 6.3
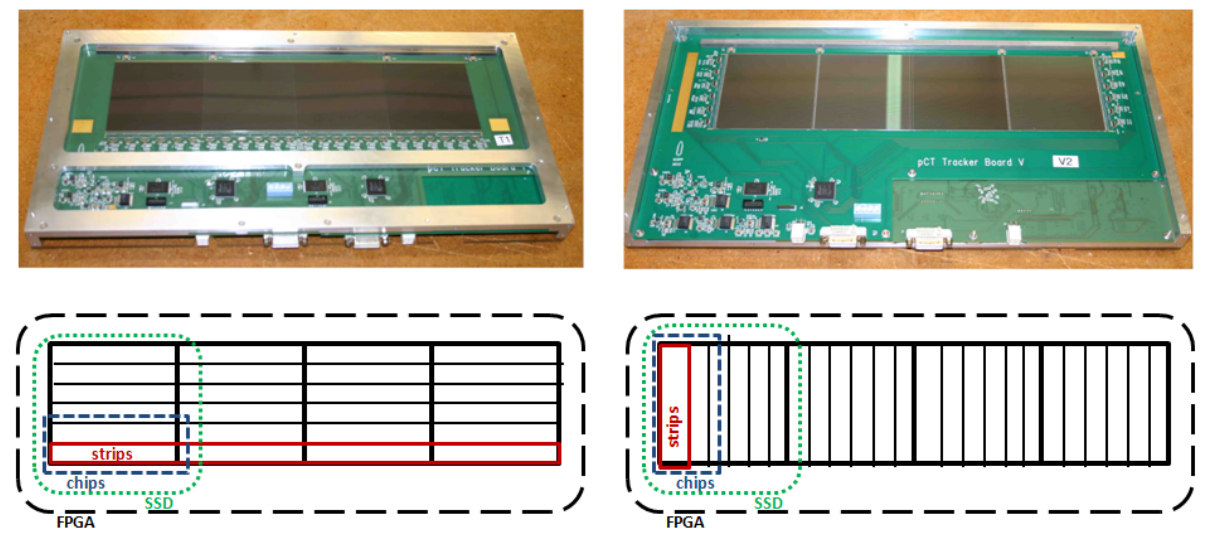

Figure 2: (top row) Silicon strip detector (SSD) plane, front and rear side. (bottom row) Schematic representation of strips, chips, SSD's, FPGA's.

\subsection{Energy detector}

The energy detector of the Phase-II pCT scanner was designed for optimal WEPL measurement resolution 23. It comprises five polystyrene scintillator stages, each with a thickness of $51 \mathrm{~mm}$ and a lateral area of $10 \times 40 \mathrm{~cm}^{2}$. The system was modelled in detail with Geant4 by Giacometti et al. 105. The optical reflectors and the photomultiplier tubes were modelled only as passive elements, since optical photons were not explicitly simulated.

The quantity registered in the energy detector during simulation is the total energy deposit in a stage during an event (one primary proton), not taking into account pile-up. That level of detail in the modelling of the detection principle 
allowed to simulate the basic detector effects and reproduced to a large extent the experimental pCT images 105. However, in order to understand the origin of artifacts in images acquired with the Phase-II pCT scanner, it was necessary to simulate and/or parameterize the whole detection and digitization procedure of the energy detector as accurately as possible. To this end, a few additional steps were taken by Dickmann et al. 42, and are briefly summarized here.

${ }_{545}$ The light production in the scintillators caused by high-LET particles, such as protons of low energy, becomes a non-linear function of the deposited energy, due to the quenching (Birks') effect [106]. As optical photons are not transported, the simulation needs to account for this non-linearity by modifying the energy deposit according to the LET of the particle causing it and the empirical Birks coefficient $k_{\mathrm{B}}$. The latter was determined by fitting Birks' law to experimental data 42 .

The signal produced by a scintillator stage is also dependent on the location of the energy deposit with respect to the photomultiplier. Without explicit optical photon simulation, the effect was parameterized with the use of an analytical formula derived from experimental data. According to that parameterization, the energy deposit registered was modified and resulted in what was called the $\mathrm{TV}$ effect (from the dependence of the signal on horizontal $t$ and vertical $v$ position) [23. The spatially dependent energy deposit was transformed into analog-to-digital converter (ADC) counts to which noise and an offset (called pedestal) derived from experimental data were added. Finally, the energy detector data are combined with the tracker data and written out in the exact same format as the experimental raw data. This allows for the postprocessing of simulated and experimental data interchangeably, as detailed in the following section.

\subsection{Postprocessing}

Processing of the raw data and detector calibration may have a large impact on the reconstructed $\mathrm{pCT}$ image and are therefore an indispensable part of a fully realistic pCT scanner simulation chain.

For the Phase-II pCT scanner, a two-step calibration procedure is required to map ADC counts output from the scanner 20 (or the MC simulation) to absolute measurements of the energy deposit, and eventually to WEPL values for every proton.

The first calibration step employs protons acquired without an object. For every detector stage, a histogram is made of measured ADC counts. It generally 575 exhibits two distinct peaks: one full-energy peak and a smaller peak corresponding to zero-energy deposits called the pedestal. The expected energy deposit of the full-energy peak can be estimated using a MC simulation, ideally accounting for the non-linear response of the scintillator due to the Birks' effect 42. This, allows to establish a linear relationship between ADC count and an absolute measurement of the energy deposit in each stage

$$
E(a)=g \cdot(a-p)
$$


where $E$ is the absolute energy measurement corresponding to the ADC value $a, p$ is the pedestal (the ADC value of the zero-energy-peak) and $g$ is the gain.

Due to the geometric shape of the detector, the TV effects described in section 6.2 make this calibration depend on the hit location of the proton within the energy detector. To account for this, the stage hit coordinates $t$ and $v$ are estimated from tracking information, and proton ADC counts are stored in bins based on a spatial grid with the size of $10 \mathrm{~mm}$ by $10 \mathrm{~mm}$ in coordinates $t$ and $v$. At each point of the grid, $g=g(t, v)$ is estimated and stored in a lookup-table, allowing for a correction of the TV effect in subsequent runs.

The second calibration step requires five data sets taken using a doublewedge-shaped calibration phantom made from polystyrene, together with zero to four $51 \mathrm{~mm}$ thick blocks of the same material. Combination of the wedge and blocks cover the entire WEPL range of the detector: from $0 \mathrm{~mm}$ to $255 \mathrm{~mm}$. Both the exact geometry and the RSP of the calibration phantom are known. For every proton, the correct WEPL can be calculated from its trajectory as measured by tracking, together with the known geometry of the calibration phantom. Additionally, the stage in which it stops is assumed to be the furthest stage with a calibrated energy deposit above $1 \mathrm{MeV}$. For a given stopping stage, proton energies are sorted into bins of $0.25 \mathrm{MeV}$ width according to their mea-

600 sured energy deposit in that stage. For each of these energy bins, a histogram of known WEPLs is accumulated and the most likely WEPL is estimated. Five calibration curves (one for each stage) that map the energy deposit in the stopping stage to the proton's WEPL are then created. To process an incident proton's data from a subsequent scan, first its five absolute energy deposits are calculated using eq. (1). Then, the stopping stage is determined, and using the corresponding calibration curve, the proton's WEPL is calculated.

\subsection{Phantoms}

In the case of the simulation of the Phase-II pCT scanner in Geant4, simulation of voxelised phantoms was based on DICOM extended example, which starting from DICOM images of phantoms or patients, converts them into voxelised geometries, typically by employing a stoichiometric calibration from CT values to stopping power. A high-resolution pediatric head phantom 107] was scanned and reconstructed using the Phase-II pCT scanner [105]. It is based on an existing commercially available tissue equivalent pediatric head phantom ${ }_{615}$ (model HN715, CIRS, Norfolk, Virginia, USA), and was created by merging eight separate high-resolution helical x-ray CT scans of the physical phantom (pixel size $0.1875 \mathrm{~mm} \times 0.1875 \mathrm{~mm}$ ). A segmentation process was employed to identify each of the tissue-equivalent phantom materials and assign them a unique $\mathrm{CT}$ number. This feature was exploited in Geant 4 where each CT 620 number was directly assigned to the corresponding material, thus bypassing the use of a stoichiometric calibration curve and guaranteeing a correct HU-density assignation in the simulation. Using this phantom, the agreement between simulated and experimental reconstructed relative stopping power was found to be within $1.5 \%$ [107. 


\subsection{Beam modelling}

Beam phase spaces can either be generated using assumptions on distributions of the variables or by employing a $\mathrm{MC}$ simulation of a proton beam line, including all beam optics. While the latter is the most realistic option, it requires potentially proprietary knowledge about a commercial beam line [108]. It also limits the applicability of a study to a certain beam line. Therefore, most idealized studies employ a spatially homogeneous distributions of parallel protons at a fixed energy of typically $200 \mathrm{MeV}$ [23, 109, 105, 65].

Piersimoni et al. [110] employed an incident Gaussian proton spot and included in their MC simulation scattering foils as well as magnetic fields sweeping the scattered pencil beam. They fine-tuned and validated model-parameters such as the incident proton energy spread and the initial spot size by performing measurements on the magnetic fields as well as on the resulting proton beam using (arrays of) ionization chambers. They did not disclose parameters of their model, but reported that the impact on RSP accuracy in comparison to a pure parallel proton beam, is negligible.

When investigating proton $\mathrm{CT}$ image noise, where an exact model of the fluence field is required to make simulations comparable to experiments, Dickmann et al. 42 generated a phase space from experimental tracking data, reproducing every tracked proton of a previous experimental scan in the MC simulation.

\section{MC studies based on extensive modelling}

\section{1. $p C T$ RSP accuracy}

Since the main attraction of $\mathrm{pCT}$ is its potential to reduce range uncertainties in proton therapy by improved RSP accuracy, there have been several studies relying on both MC simulation and experiments attempting to quantify this. Ideal simulation studies generally agree in stating that the achievable RSP accuracy should be much better than $1 \%$ [17, 43, 60, 108. However, experimental results for two different pCT scanners have typically slightly fallen short of the sub-percent accuracy target for at least some of the materials investigated [107, 26, 59, indicating that ideal simulations likely do not capture all effects pertinent to RSP accuracy in experimental pCT scans. To reconcile these discrepancies, MC simulations fully modelling all aspects of the pCT detection chain have been used to better understand the sources of RSP accuracy degradations. Giacometti et al. [107] used a full model of the Phase-II pCT scanner detector, however scoring only energy deposits and obtained RSP differences between MC and experiment bounded by $-1.7 \%$. Section 6.2 describes how that platform was subsequently extended to model additional detector effects and Dickmann et al. 42] obtained congruent experimental and simulated energy deposit signatures, allowing interchangeable measured and simulated input to a single postprocessing pipeline. The platform was used by Dedes et al. [59] in a comparison to DECT RSP accuracy, where simulated and measured RSPs agreed within $1.3 \%$. The mean absolute percent error (MAPE) on RSP over 13 inserts was $0.55 \%$ experimentally and $0.69 \%$ based on the simulations, which 

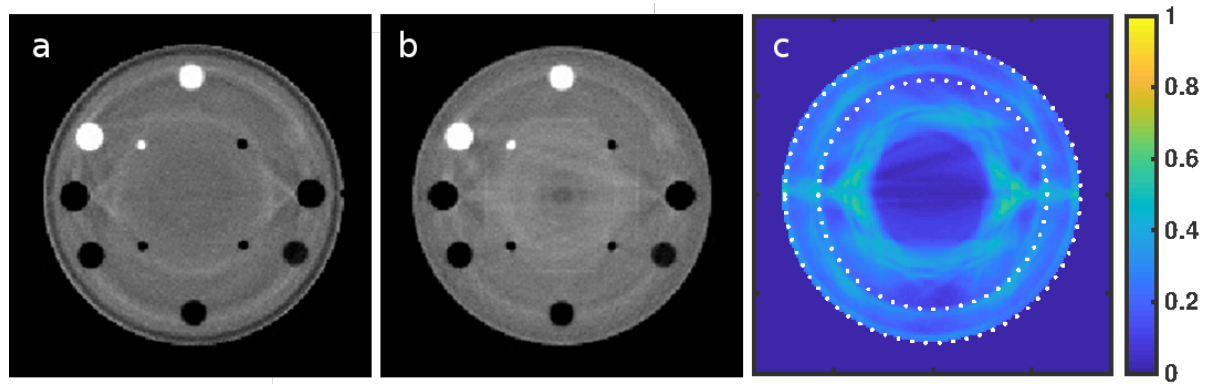

Figure 3: RSP images of module CTP404 of a Catphan phantom reconstructed from (a) measured and (b) simulated pCT acquisitions. An RSP level of 1.15 and window of 0.3 were applied on the images in order to highlight the pCT image artifacts. (c) Represents the fraction of protons stemming from areas where problematic WEPLs are expected and stands for artefacts in (a) and (b). Adapted from Dedes et al. [59. (c) Institute of Physics and Engineering in Medicine. Reproduced by permission of IOP Publishing. All rights reserved.

reproduced the location of accuracy-degrading artifacts well (see Section 7.3). The study concluded that both DECT and pCT yield sub-percent MAPE.

\section{2. $p C T$ spatial resolution}

Few MC studies dedicated to spatial resolution are reported in the literature which extensively model a pCT scanner system. The work of Plautz et al. [111 compared simulation and experimental results using a dedicated phantom for the Phase-II pCT scanner. In their simulation, tracking detectors were

675 physically present and thus contributed to scattering of protons, but tracking itself was ideal in the sense that proton positions and directions were perfectly recorded by the simulation. To mimic the uncertainty due to the finite strip pitch, tracking data were polluted afterwards with Gaussian noise. Spatial resolution in simulated and experimentally acquired pCT images agreed well.

${ }_{680}$ The authors found a resolution of $0.6-0.8 \mathrm{lp} / \mathrm{mm}$ depending on the depth of interest in a $20 \mathrm{~cm}$ water-equivalent cylindrical phantom.

Penfold et al. [112 performed a series of MC simulations to support specific design choices of the Phase-II pCT scanner. They used an explicit geometrical representation of the tracking detectors with sensitive silicon strips of $228 \mu \mathrm{m}$

685 width. As part of their results, they concluded that the distance between tracker planes of one tracker pair should be no less than $6-8 \mathrm{~cm}$.

\section{3. $p C T$ image artifacts}

Several experimental and simulation studies of the Phase-II pCT scanner [13, 107, 110, 59] note the presence of artifacts in the RSP image. In Dedes

690 et al. [59], artifacts of up to $2 \%$ in RSP were reported. In homogeneous cylindrical objects, inaccuracies in specific WEPL ranges due to the detection and calibration process, result in ring artifacts, while for heterogeneous phantoms the distortions may not be directly visible in the image. 
In Dedes et al. [59, a major cause of WEPL inaccuracy was found to be study, Dickmann et al. 114 used such simulation in a fluence modulated proton CT optimization strategy.

\subsection{Ions other than protons}

Volz et al. [115] performed experimental acquisitions of helium CT with the Phase-II pCT scanner, and used realistic MC simulations to explore the impact of secondary fragments in the detector on image artifacts. Their MC simulations allowed them to design a $\Delta E-E$ filter to eliminate such fragments exploiting the characteristic correlation between the energy deposit in the stopping stage $(E)$ and in the stage prior to that $(\Delta E)$. By defining an acceptance interval around the expected dependence of $E$ and $\Delta E$, fragments can be filtered out. They tested this approach successfully on experimental data 64. Their realistic $\mathrm{MC}$ simulations, which produce energy deposits directly instead of ADC, have suggested that the $\Delta E-E$ filter may also improve pCT artifacts [65]; however

\section{Conclusion}

In this review article, we have presented an overview of the use of MC simulation in proton computed tomography for radiation therapy applications. As evidenced by the literature reviewed, MC simulations that omit the detection 


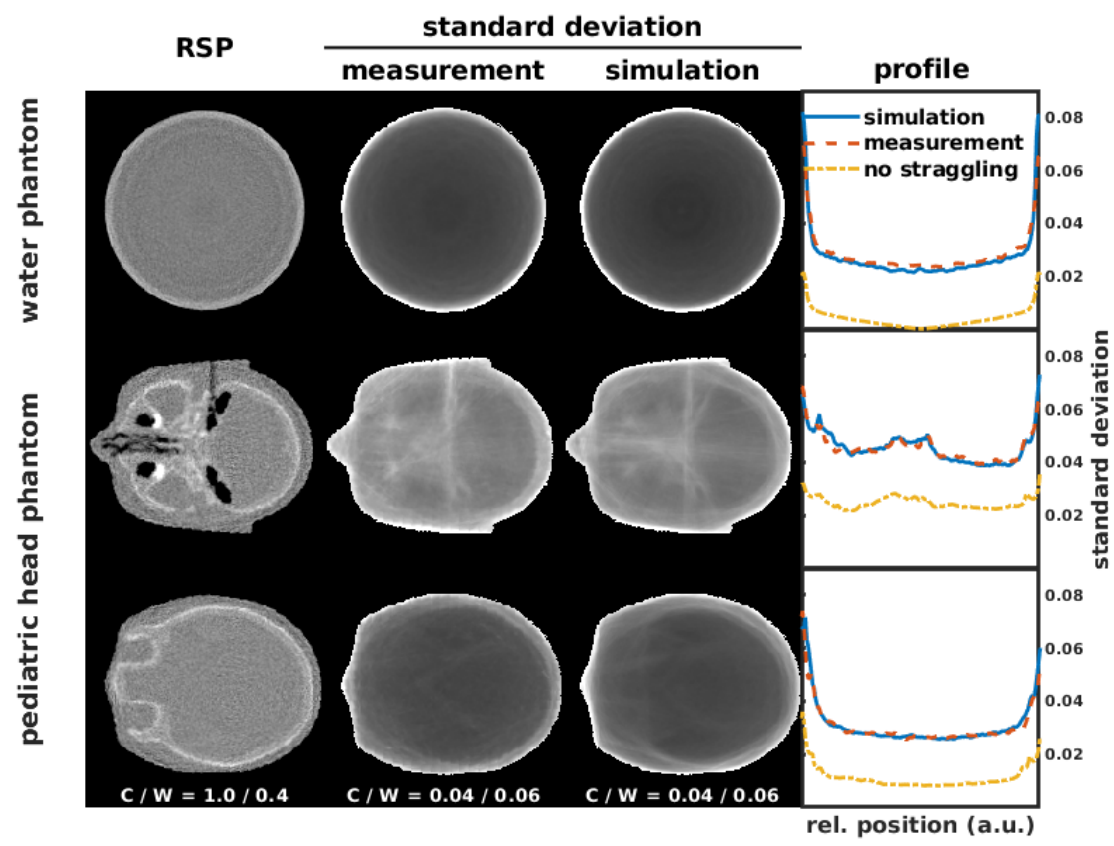

Figure 4: (left column) The reconstructed RSP images from experimental pCT scans of (top row) a water cylinder and (bottom two rows) a pediatric head phantom obtained from the Phase-II pCT scanner. (two central columns) The corresponding noise reconstructions are shown for measurements and corresponding Monte Carlo simulations, along with (right col$u m n$ ) horizontal noise profiles spanning the object edge to edge; cummulative noise from experimental and simulated acquisitions is plotted in addition to the noise expected without energy straggling (scattering noise). Reproduced from Dickmann et al. 42 under CC BY 3.0 license.

process are useful tools for exploring the feasibility, the theoretical limits and the potential of pCT. Several studies have assessed the maximum RSP accuracy and precision as well as the spatial resolution and the biological impact of imaging dose. With a few pCT prototype scanners being operational or in development, recent research has focused on optimizing, understanding and utilizing pCT scanners in order to approach the theoretical limits of pCT image quality and dose efficiency. In this context, fully detailed MC simulations can provide key insight on the impact of the detection system. We presented the example of modelling the detection process of an existing prototype scanner, extensively validated with experimental $\mathrm{pCT}$ acquisitions. The use of such a 745 detailed MC simulation platform, in combination with measured data, led to deeper understanding of the origin of image artifacts, noise patterns and dose efficiency of pCT, answering some of the open questions en route to a clinically applicable pCT system. 


\section{Acknowledgements}

The authors acknowledge support from the German Research Foundation (DFG Project numbers: 388731804 and 372393016) and the Bavaria California Technology Center (BaCaTeC project numbers: 28 [2015-2] and A1 [2018-2]). The work of Nils Krah was partially supported by the project DIC20161236452 of the Fondation pour la Recherche Médicale. The authors would additionally like to thank Mark Pankuch for discussions on proton beamline characteristics.

\section{References}

[1] A. M. Cormack, Representation of a Function by Its Line Integrals, with Some Radiological Applications, Journal of Applied Physics 34 (1963) 2722-2727. URL: http://aip.scitation.org/doi/10.1063/1. 1713127http://aip.scitation.org/doi/10.1063/1.1729798 doi/10. 1063/1.1729798.

[2] A. M. Cormack, Representation of a Function by Its Line Integrals, with Some Radiological Applications. II, Journal of Applied Physics 35 (1964) 2908-2913. URL: http://aip.scitation.org/doi/10.1063/ 1.1713127, doi $10.1063 / 1.1713127$.

[3] A. M. Koehler, Proton Radiography, Science 160 (1968) 303 LP 304. URL: http://science.sciencemag.org/content/160/3825/303. abstracthttp://www.sciencemag.org/cgi/doi/10.1126/science. 160.3825.303, doi $10.1126 /$ science.160.3825.303.

[4] A. M. Cormack, A. M. Koehler, Quantitative proton tomography: preliminary experiments, Physics in Medicine and Biology 21 (1976) 007. URL: http://stacks.iop.org/0031-9155/21/i= 4/a=007?key=crossref .88d730914cc828b5a49bdf617ede881f doi 10 . $1088 / 0031-9155 / 21 / 4 / 007$

[5] K. M. Hanson, J. N. Bradbury, T. M. Cannon, R. L. Hutson, D. B. Laubacher, R. Macek, M. A. Paciotti, C. A. Taylor, Application of protons to computer tomography, Technical Report, 1977.

[6] K. M. Hanson, Proton computed tomography, IEEE Transactions on Nuclear Science 26 (1979) 1635-1640.

[7] K. M. Hanson, J. N. Bradbury, T. M. Cannon, R. L. Hutson, D. B. Laubacher, R. J. MacEk, M. A. Paciotti, C. A. Taylor, Computed tomography using proton energy loss, Physics in Medicine and Biology 26 (1981) 965-983. doi 10.1088/0031-9155/26/6/001.

[8] K. M. Hanson, J. N. Bradbury, R. A. Koeppe, R. J. MacEk, D. R. MacHen, R. Morgado, M. A. Paciotti, S. A. Sandford, V. W. Steward, Proton computed tomography of human specimens, Physics in Medicine and Biology 27 (1982) 25-36. doi 10.1088/0031-9155/27/1/003. 
[9] R. W. Schulte, S. N. Penfold, Proton CT for improved stopping power determination in proton therapy, Transactions of the American Nuclear Society 106 (2012) 55-58.

[10] G. Poludniowski, N. M. Allinson, P. M. Evans, Proton radiography and tomography with application to proton therapy, The British Journal of Radiology 88 (2015) 20150134. URL: http://www.birpublications.org/ doi/10.1259/bjr.20150134 doi $10.1259 /$ bjr.20150134

[17] D. C. Hansen, N. Bassler, T. S. Sørensen, J. Seco, The image quality of ion computed tomography at clinical imaging dose levels, Medical physics 41 (2014) 111908.

[18] S. Rit, G. Dedes, N. Freud, D. Sarrut, J. M. Létang, Filtered backprojection proton CT reconstruction along most likely paths, Medical Physics 40 (2013) 031103. URL: http://doi.wiley.com/10.1118/1.4789589 doi $10.1118 / 1.4789589$.

825 [19] G. Poludniowski, N. M. Allinson, P. M. Evans, Proton computed tomography reconstruction using a backprojection-thenfiltering approach, Physics in Medicine and Biology 59 (2014) 


1905-7918. URL: http://stacks.iop.org/0031-9155/59/i=
24/a=7905?key=crossref .ba56732f6b55c3c7e9a73e19c2be9417.
doi 10.1088/0031-9155/59/24/7905.

[20] R. P. Johnson, V. Bashkirov, L. DeWitt, V. Giacometti, R. F. Hurley, P. Piersimoni, T. E. Plautz, H. F. Sadrozinski, K. Schubert, R. Schulte, B. Schultze, A. Zatserklyaniy, A Fast Experimental Scanner for Proton CT: Technical Performance and First Experience With Phantom Scans, IEEE Transactions on Nuclear Science 63 (2016) 52-60. URL: http://ieeexplore.ieee.org/document/7352382/, doi:10.1109/TNS. 2015.2491918.

[21] H. Paganetti, Range uncertainties in proton therapy and the role of Monte Carlo simulations, 2012. doi:10.1088/0031-9155/57/11/R99.

${ }_{840}[22]$ S. B. Crowe, B. Sutherland, R. Wilks, V. Seshadri, S. Sylvander, J. V. Trapp, T. Kairn, Technical Note: Relationships between gamma criteria and action levels: Results of a multicenter audit of gamma agree-

口 ment index results, Medical Physics 43 (2016) 1501-1506. doi 10.1118/ 1.4942488

${ }_{845}[23]$ V. A. Bashkirov, R. W. Schulte, R. F. Hurley, R. P. Johnson, H. F.-W. Sadrozinski, A. Zatserklyaniy, T. Plautz, V. Giacometti, Novel scintillation detector design and performance for proton radiography and computed tomography, Medical Physics 43 (2016) 664674. URL:http://dx.doi.org/10.1118/1.4939255http://doi.wiley. com/10.1118/1.4939255, doi:10.1118/1.4939255.

[24] C. Miller, B. Altoos, E. A. DeJongh, M. Pankuch, D. F. DeJongh, V. Rykalin, C. E. Ordoñez, N. T. Karonis, J. R. Winans, G. Coutrakon, J. S. Welsh, Reconstructed and real proton radiographs for image-guidance in proton beam therapy, Journal of Radiation Oncology 8 (2019) 97-101. URL: http://link.springer.com/10.1007/ s13566-019-00376-0, doi:10.1007/s13566-019-00376-0.

[25] J. F. Janni, Energy loss, range, path length, time-of-flight, straggling, multiple scattering, and nuclear interaction probability, Atomic Data and Nuclear Data Tables (1982). doi 10.1016/0092-640x(82)90005-5.

[26] M. Esposito, C. Waltham, J. T. Taylor, S. Manger, B. Phoenix, T. Price, G. Poludniowski, S. Green, P. M. Evans, P. P. Allport, S. Manolopulos, J. Nieto-Camero, J. Symons, N. M. Allinson, PRaVDA: The first solid-state system for proton computed tomography, Physica Medica 55

1. (2018) 149-154. URL: https://linkinghub.elsevier.com/retrieve/ pii/S1120179718313073, doi:10.1016/j.ejmp.2018.10.020

[27] H. E. S. Pettersen, J. Alme, G. G. Barnaföldi, R. Barthel, A. van den Brink, M. Chaar, V. Eikeland, A. García-Santos, G. Genov, S. Grimstad, O. Grøttvik, H. Helstrup, K. F. Hetland, S. Mehendale, I. Meric, 
O. H. Odland, G. Papp, T. Peitzmann, P. Piersimoni, A. Ur Rehman, M. Richter, A. T. Samnøy, J. Seco, H. Shafiee, E. V. Skjæveland, J. R. Sølie, G. Tambave, K. Ullaland, M. Varga-Kofarago, L. Volz, B. Wagner, S. Yang, D. Röhrich, Design optimization of a pixel-based range telescope for proton computed tomography, Physica Medica 63 (2019) 87-97. URL: https://doi.org/10.1016/j.ejmp.2019.05.026https: //linkinghub.elsevier.com/retrieve/pii/S1120179719301358. doi $10.1016 / j$.ejmp. 2019.05.026.

[28] S. Agostinelli, J. Allison, K. Amako, J. Apostolakis, H. Araujo, P. Arce, M. Asai, D. Axen, S. Banerjee, G. Barrand, F. Behner, L. Bellagamba, J. Boudreau, L. Broglia, A. Brunengo, H. Burkhardt, S. Chauvie, J. Chuma, R. Chytracek, G. Cooperman, G. Cosmo, P. Degtyarenko, A. Dell'Acqua, G. Depaola, D. Dietrich, R. Enami, A. Feliciello, C. Ferguson, H. Fesefeldt, G. Folger, F. Foppiano, A. Forti, S. Garelli, S. Giani, R. Giannitrapani, D. Gibin, J. Gómez Cadenas, I. González, G. Gracia Abril, G. Greeniaus, W. Greiner, V. Grichine, A. Grossheim, S. Guatelli, P. Gumplinger, R. Hamatsu, K. Hashimoto, H. Hasui, A. Heikkinen, A. Howard, V. Ivanchenko, A. Johnson, F. Jones, J. Kallenbach, N. Kanaya, M. Kawabata, Y. Kawabata, M. Kawaguti, S. Kelner, P. Kent, A. Kimura, T. Kodama, R. Kokoulin, M. Kossov, H. Kurashige, E. Lamanna, T. Lampén, V. Lara, V. Lefebure, F. Lei, M. Liendl, W. Lockman, F. Longo, S. Magni, M. Maire, E. Medernach, K. Minamimoto, P. Mora de Freitas, Y. Morita, K. Murakami, M. Nagamatu, R. Nartallo, P. Nieminen, T. Nishimura, K. Ohtsubo, M. Okamura, S. O'Neale, Y. Oohata, K. Paech, J. Perl, A. Pfeiffer, M. Pia, F. Ranjard, A. Rybin, S. Sadilov, E. Di Salvo, G. Santin, T. Sasaki, N. Savvas, Y. Sawada, S. Scherer, S. Sei, V. Sirotenko, D. Smith, N. Starkov, H. Stoecker, J. Sulkimo, M. Takahata, S. Tanaka, E. Tcherniaev, E. Safai Tehrani, M. Tropeano, P. Truscott, H. Uno, L. Urban, P. Urban, M. Verderi, A. Walkden, W. Wander, H. Weber, J. Wellisch, T. Wenaus, D. Williams, D. Wright, T. Yamada, H. Yoshida, D. Zschiesche, Geant4 - a simulation toolkit, Nuclear Instruments and Methods in Physics Research Section A: Accelerators, Spectrometers, Detectors and Associated Equipment 506 (2003) 250-303. URL: http://linkinghub.elsevier.com/retrieve/ pii/S0168900203013688, doi:10.1016/S0168-9002(03)01368-8.

[29] A. Ferrari, P. R. Sala, A. Fassò, J. Ranft, FLUKA: a multi-particle transport code, Technical Report, CERN-2005-10 (2005), INFN/TC 05/11, SLAC-R-773, 2005.

[30] S. Jan, G. Santin, D. Strul, S. Staelens, K. Assié, D. Autret, S. Avner, R. Barbier, M. Bardiès, P. M. Bloomfield, D. Brasse, V. Breton, P. Bruyndonckx, I. Buvat, A. F. Chatziioannou, Y. Choi, Y. H. Chung, C. Comtat, D. Donnarieix, L. Ferrer, S. J. Glick, C. J. Groiselle, D. Guez, P. F. Honore, S. Kerhoas-Cavata, A. S. Kirov, V. Kohli, M. Koole, M. Krieguer, D. J. van der Laan, F. Lamare, G. Largeron, C. Lartizien, D. Lazaro, 
M. C. Maas, L. Maigne, F. Mayet, F. Melot, C. Merheb, E. Pennacchio, J. Perez, U. Pietrzyk, F. R. Rannou, M. Rey, D. R. Schaart, C. R. Schmidtlein, L. Simon, T. Y. Song, J. M. Vieira, D. Visvikis, R. Van de Walle, E. Wieërs, C. Morel, GATE: A simulation toolkit for PET and SPECT, Physics in Medicine and Biology 49 (2004) 4543-4561. doi $10.1088 / 0031-9155 / 49 / 19 / 007$.

[31] J. Perl, J. Shin, J. Schümann, B. Faddegon, H. Paganetti, TOPAS: An innovative proton Monte Carlo platform for research and clinical applications, Medical Physics 39 (2012) 6818-6837. doi:10.1118/1.4758060.

[32] T. T. Böhlen, F. Cerutti, M. Dosanjh, A. Ferrari, I. Gudowska, A. Mairani, J. M. Quesada, Benchmarking nuclear models of FLUKA and GEANT4 for carbon ion therapy, Physics in Medicine and Biology (2010). doi:10.

[33] L. Grevillot, D. Bertrand, F. Dessy, N. Freud, D. Sarrut, GATE as a GEANT4-based Monte Carlo platform for the evaluation of proton pencil beam scanning treatment plans, Physics in Medicine and Biology (2012). doi $10.1088 / 0031-9155 / 57 / 13 / 4223$.

[34] C. Robert, G. Dedes, G. Battistoni, T. T. Böhlen, I. Buvat, F. Cerutti, M. P. Chin, A. Ferrari, P. Gueth, C. Kurz, L. Lestand, A. Mairani, G. Montarou, R. Nicolini, P. G. Ortega, K. Parodi, Y. Prezado, P. R. Sala, D. Sarrut, E. Testa, Distributions of secondary particles in proton and carbon-ion therapy: A comparison between GATE/Geant4 and FLUKA Monte Carlo codes, Physics in Medicine and Biology (2013). doi $10.1088 / 0031-9155 / 58 / 9 / 2879$

[35] M. Testa, J. Schümann, H. M. Lu, J. Shin, B. Faddegon, J. Perl, H. Paganetti, Experimental validation of the TOPAS Monte Carlo system for passive scattering proton therapy, Medical Physics (2013). doi $10.1118 / 1.4828781$.

[36] G. Battistoni, J. Bauer, T. T. Boehlen, F. Cerutti, M. P. Chin, R. Dos Santos Augusto, A. Ferrari, P. G. Ortega, W. Kozlowska, G. Magro, A. Mairani, K. Parodi, P. R. Sala, P. Schoofs, T. Tessonnier, V. Vlachoudis, The FLUKA code: An accurate simulation tool for particle therapy, Frontiers in Oncology (2016). doi 10.3389/fonc.2016.00116.

[37] K. S. Baumann, F. Horst, K. Zink, C. Gomà, Comparison of penh, fluka, and Geant4/topas for absorbed dose calculations in air cavities representing ionization chambers in high-energy photon and proton beams, Medical Physics (2019). doi:10.1002/mp.13737.

[38] W. S. Kozłowska, T. T. Böhlen, C. Cuccagna, A. Ferrari, F. Fracchiolla, G. Magro, A. Mairani, M. Schwarz, V. Vlachoudis, D. Georg, FLUKA particle therapy tool for Monte Carlo independent calculation of scanned 
proton and carbon ion beam therapy, Physics in Medicine and Biology (2019). doi:10.1088/1361-6560/ab02cb

[39] C. Bopp, J. Colin, D. Cussol, C. Finck, M. Labalme, M. Rousseau, D. Brasse, Proton computed tomography from multiple physics pron cesses, Physics in Medicine and Biology 58 (2013) 7261-7276. doi 10. 1088/0031-9155/58/20/7261.

[40] C. T. Quiñones, J. M. Létang, S. Rit, Filtered back-projection reconstruction for attenuation proton CT along most likely paths, Physics in Medicine and Biology 61 (2016) 3258-3278. doi:10.1088/0031-9155/61/ 9/3258.

[41] J. T. Taylor, G. Poludniowski, T. Price, C. Waltham, P. P. Allport, G. L. Casse, M. Esposito, P. M. Evans, S. Green, S. Manger, S. Manolopoulos, J. Nieto-Camero, D. J. Parker, J. Symons, N. M. Allinson, An experimental demonstration of a new type of proton computed tomography using a novel silicon tracking detector, Medical Physics 43 (2016) 6129-6136. doi $10.1118 / 1.4965809$.

[42] J. Dickmann, P. Wesp, M. Rädler, S. Rit, M. Pankuch, R. P. Johnson, V. Bashkirov, R. W. Schulte, K. Parodi, G. Landry, G. Dedes, Prediction of image noise contributions in proton computed tomography and comparison to measurements, Physics in Medicine \& Biology 64 (2019) 145016. doi $10.1088 / 1361-6560 / a b 2474$.

[43] D. C. Hansen, J. Seco, T. S. Sørensen, J. B. B. Petersen, J. E. Wildberger, F. Verhaegen, G. Landry, A simulation study on proton computed tomography $(\mathrm{CT})$ stopping power accuracy using dual energy CT scans as benchmark, Acta Oncologica 54 (2015) 1638-1642. URL: http://www.tandfonline.com/doi/full/10.3109/ 0284186X.2015.1061212, doi 10.3109/0284186X.2015.1061212.

[44] S. Meyer, F. Kamp, T. Tessonnier, A. Mairani, C. Belka, D. J. Carlson, C. Gianoli, K. Parodi, Dosimetric accuracy and radiobiological implications of ion computed tomography for proton therapy treatment planning, Physics in Medicine and Biology (2019). URL: http: //iopscience.iop.org/article/10.1088/1361-6560/ab0fdf. doi/10. 1088/1361-6560/ab0fdf.

[45] D. Wang, T. R. MacKie, W. A. Tomé, On the use of a proton path probability map for proton computed tomography reconstruction, Medical Physics 37 (2010) 4138-4145. doi:10.1118/1.3453767.

[46] T. Li, Z. Liang, J. V. Singanallur, T. J. Satogata, D. C. Williams, R. W. Schulte, Reconstruction for proton computed tomography by tracing proton trajectories: A Monte Carlo study, Medical Physics 33 (2006) 699-706. doi $10.1118 / 1.2171507$. 
[47] S. N. Penfold, R. W. Schulte, Y. Censor, V. Bashkirov, A. B. Rosenfeld, Characteristics of proton CT images reconstructed with filtered backprojection and iterative projection algorithms, in: IEEE Nuclear Science Symposium Conference Record, 2009. doi:10.1109/NSSMIC.2009. 5402282 .

[48] N. T. Karonis, K. L. Duffin, C. E. Ordoñez, B. Erdelyi, T. D. Uram, E. C. Olson, G. Coutrakon, M. E. Papka, Distributed and hardware accelerated computing for clinical medical imaging using proton computed tomography (pCT), Journal of Parallel and Distributed Computing 73 (2013) 1605-1612. URL: http://dx.doi.org/10.1016/j.jpdc.2013. 07.016. doi $10.1016 / j \cdot j p d c .2013 .07 .016$

[49] D. C. Hansen, T. Sangild Sørensen, S. Rit, Fast reconstruction of low dose proton CT by sinogram interpolation, Physics in Medicine and Biology 61 (2016) 5868-5882. URL: http://stacks.iop.org/0031-9155/61/ $i=15 / a=5868$ ?key=crossref $.3 d 84339 \mathrm{bf} 8 \mathrm{~b} 1 \mathrm{a} 45 \mathrm{e} 6 \mathrm{eae} 572 \mathrm{f} 6 \mathrm{~b} 546 \mathrm{ee} 0$. doi $10.1088 / 0031-9155 / 61 / 15 / 5868$.

[50] D. C. Hansen, J. B. B. Petersen, N. Bassler, T. S. Sørensen, Improved proton computed tomography by dual modality image reconstruction, Medical Physics 41 (2014). doi $10.1118 / 1.4864239$.

[51] S. N. Penfold, Image reconstruction and Monte Carlo simulations in the development of proton computed tomography for applications in proton radiation therapy, Thesis (2010).

1015 [52] D. Wang, T. R. MacKie, W. A. Tomé, On proton CT reconstruction using MVCT-converted virtual proton projections, Medical Physics 39 (2012) 2997-3008. doi $10.1118 / 1.4711752$.

[53] G. A. Cirrone, M. Bucciolini, M. Bruzzi, G. Candiano, C. Civinini, G. Cuttone, P. Guarino, D. Lo Presti, S. E. Mazzaglia, S. Pallotta, N. Randazzo, 1020 V. Sipala, C. Stancampiano, C. Talamonti, Monte Carlo evaluation of the Filtered Back Projection method for image reconstruction in proton computed tomography, Nuclear Instruments and Methods in Physics Research, Section A: Accelerators, Spectrometers, Detectors and Associ$1025 \quad$ ated Equipment 658 (2011) 78-83. URL: http://dx.doi.org/10.1016/

[54] V. Sipala, M. Bruzzi, M. Bucciolini, M. Carpinelli, G. A. Cirrone, C. Civinini, G. Cuttone, D. Lo Presti, S. Pallotta, C. Pugliatti, N. Randazzo, F. Romano, M. Scaringella, C. Stancampiano, C. Talamonti, M. Tesi, E. Vanzi, M. Zani, A proton computed tomography system for medical applications, Journal of Instrumentation 8 (2013). doi $10.1088 / 1748-0221 / 8 / 02 / \mathrm{C0} 2021$. 
[55] C.-A. Collins-Fekete, S. Brousmiche, S. K. N. Portillo, L. Beaulieu, J. Seco, A maximum likelihood method for high resolution proton radiography/proton CT, Physics in Medicine and Biology 61 (2016) 8232-8248. URL: http://stacks.iop.org/0031-9155/61/ $i=23 / a=8232$ ?key=crossref $.3714 c d 4$ e1e67ee0be67c7edf $3 f 01$ e35a. doi $10.1088 / 0031-9155 / 61 / 23 / 8232$.

[56] F. Khellaf, N. Krah, J. M. Létang, S. Rit, 2D directional ramp filter, Physics in Medicine \& Biology 65 (2020) 08NT01. URL: https: //iopscience.iop.org/article/10.1088/1361-6560/ab7875, doi:10. 1088/1361-6560/ab7875.

[57] F. Khellaf, N. Krah, J.-M. Letang, C.-A. Collins-Fekete, S. Rit, A comparison of direct reconstruction algorithms in proton computed 1 tomography, Physics in Medicine \& Biology (2020). URL: https: //iopscience.iop.org/article/10.1088/1361-6560/ab7d53, doi/10. 1088/1361-6560/ab7d53.

[58] R. W. Schulte, V. Bashkirov, M. C. Klock, T. Li, A. J. Wroe, I. Evseev, D. C. Williams, T. Satogata, Density resolution of proton computed 1050 tomography, Medical Physics 32 (2005) 1035-1046. doi 10.1118/1.

[59] G. Dedes, J. Dickmann, K. Niepel, P. Wesp, R. P. Johnson, M. Pankuch, V. Bashkirov, S. Rit, L. Volz, R. W. Schulte, G. Landry, K. Parodi, Experimental comparison of proton $\mathrm{CT}$ and dual energy x-ray CT for relative stopping power estimation in proton therapy, Physics in Medicine \& Biology 64 (2019) 165002. URL: https://iopscience.iop.org/article/ 10.1088/1361-6560/ab2b72, doi 10.1088/1361-6560/ab2b72.

[60] N. Arbor, D. Dauvergne, G. Dedes, J. M. Létang, K. Parodi, C. T. Quiñones, E. Testa, S. Rit, Monte Carlo comparison of $\mathrm{x}$-ray and proton $\mathrm{CT}$ for range calculations of proton therapy beams, Physics in Medicine and Biology 60 (2015)

1 7585-7599. URL: http://stacks.iop.org/0031-9155/60/i= $19 / \mathrm{a}=7585$ ?key=crossref $.57 \mathrm{a} 1486 \mathrm{c} 25 \mathrm{f} 9 \mathrm{eea} 1404 \mathrm{e} 24 \mathrm{c} 3 \mathrm{be} 3 \mathrm{deb} 7 \mathrm{c}$ doi $10.1088 / 0031-9155 / 60 / 19 / 7585$.

[61] M. Rädler, G. Landry, S. Rit, R. W. Schulte, K. Parodi, G. Dedes, Twodimensional noise reconstruction in proton computed tomography using distance-driven filtered back-projection of simulated projections, Physics in Medicine \& Biology 63 (2018) 215009. URL: http://iopscience.iop. org/10.1088/1361-6560/aae5c9, doi:10.1088/1361-6560/aae5c9.

[62] C.-A. Collins-Fekete, N. Dikaios, G. Royle, P. M. Evans, Statisti1070 cal limitations in proton imaging, Physics in Medicine \& Biology 65

(1) (2020) 085011. URL: https://iopscience.iop.org/article/10.1088/ 1361-6560/ab7972, doi:10.1088/1361-6560/ab7972. 
[63] P. Piersimoni, B. A. Faddegon, J. R. Méndez, R. W. Schulte, L. Volz, J. Seco, Helium CT: Monte Carlo simulation results for an ideal source and detector with comparison to proton CT, Medical Physics 45 (2018) 3264-

11 3274. URL: https://onlinelibrary.wiley.com/doi/abs/10.1002/mp. 12942, doi $10.1002 / \mathrm{mp} .12942$.

[64] L. Volz, P. Piersimoni, V. A. Bashkirov, S. Brons, C.-A. Collins-Fekete, R. P. Johnson, R. W. Schulte, J. Seco, The impact of secondary fragments on the image quality of helium ion imaging, Physics in Medicine and

Biology 63 (2018) 195016. URL: http://iopscience.iop.org/article/ 10.1088/1361-6560/aadf25http://stacks.iop.org/0031-9155/63/ $i=19 / a=195016$ ?key=crossref $.97 c 973 c 4942 d c 76 f 6137118 f c d 32 f d 5 b$. doi $10.1088 / 1361-6560 /$ aadf25.

[65] L. Volz, P. Piersimoni, R. P. Johnson, V. A. Bashkirov, R. W. Schulte, J. Seco, Improving single-event proton CT by removing nuclear interaction events within the energy/range detector, Physics in Medicine \& Biology 64 (2019) 15NT01. doi 10.1088/1361-6560/ab2671.

[66] D. C. Williams, The most likely path of an energetic charged particle through a uniform medium, Physics in Medicine and Biology 49 (2004) 2899-2911. URL: http://stacks.iop.org/0031-9155/49/ $i=13 / a=010$ ?key=crossref . 0d8a5a7aa400200a7b9d78ead80 f fac8. doi $10.1088 / 0031-9155 / 49 / 13 / 010$

[67] G. R. Lynch, O. I. Dahl, Approximations to multiple Coulomb scattering, Nuclear Inst. and Methods in Physics Research, B (1991). doi 10.1016/ 0168-583X (91) 95671-Y.

[68] B. Gottschalk, A. M. Koehler, R. J. Schneider, J. M. Sisterson, M. S. Wagner, Multiple Coulomb scattering of $160 \mathrm{MeV}$ protons, Nuclear Inst. and Methods in Physics Research, B (1993). doi:10.1016/0168-583X (93) 95944-Z

[69] B. Erdelyi, A comprehensive study of the most likely path formalism for proton-computed tomography, Physics in Medicine and Biology (2009). doi $10.1088 / 0031-9155 / 54 / 20 / 005$

[70] C.-A. Collins-Fekete, P. Doolan, M. F. Dias, L. Beaulieu, J. Seco, Developing a phenomenological model of the proton trajectory within a heterogeneous medium required for proton imaging, Physics in Medicine and Biology 60 (2015) 5071-5082. URL: http://stacks.iop.org/0031-9155/ $60 / i=13 / a=5071$ ?key=crossref $.07 a 244970042$ bcf ca91c77a0348e8502. doi $10.1088 / 0031-9155 / 60 / 13 / 5071$.

1110 [71] N. Krah, J. M. Létang, S. Rit, Polynomial modelling of proton trajectories in homogeneous media for fast most likely path estimation and trajectory simulation, Physics in Medicine and Biology (2019). doi $10.1088 / 1361-6560 / a b 3 d 0 b$. 
[72] C. A. Collins-Fekete, E. Bar, L. Volz, H. Bouchard, L. Beaulieu, J. Seco, Extension of the Fermi-Eyges most-likely path in heterogeneous medium with prior knowledge information, Physics in Medicine and Biology 62 (2017) 9207-9219. doi:10.1088/1361-6560/aa955d

[73] M. D. Brooke, S. N. Penfold, An inhomogeneous most likely path formalism for proton computed tomography, Physica Medica 70 (2020) 184-195. URL: https://linkinghub.elsevier.com/retrieve/ pii/S1120179720300284 doi:10.1016/j.ejmp.2020.01.025.

[74] F. Khellaf, N. Krah, I. Rinaldi, J.-M. Létang, S. Rit, Effects of transverse heterogeneities on the most likely path of protons, Physics in Medicine and Biology (2019) 1-3. URL: http://iopscience.iop.org/article/ 10.1088/1361-6560/ab02a8 doi/10.1088/1361-6560/ab02a8

[75] A. Makarova, B. Gottschalk, W. Sauerwein, Comparison of Geant4 multiple Coulomb scattering models with theory for radiotherapy protons, Physics in Medicine and Biology (2017). doi:10.1088/1361-6560/aa6ce3.

[76] C. Bopp, R. Rescigno, M. Rousseau, D. Brasse, The impact of tracking system properties on the most likely path estimation in protonCT, Physics in Medicine and Biology 59 (2014) N197-N210. doi 10.1088/0031-9155/ $59 / 23 / \mathrm{N} 197$

[77] U. Schneider, E. Pedroni, M. Hartmann, T. Lomax, TH-E-220-02: Spatial Resolution of Proton Tomography: Methods, Initial Phase Space and Object Thickness, in: Medical Physics, 2011. doi 10.1118/1.3613609.

[78] U. Schneider, J. Besserer, M. Hartmann, Technical Note: Spatial resolution of proton tomography: Impact of air gap between patient and detector, Medical Physics 39 (2012) 798-800. doi:10.1118/1.3676739.

[79] N. Krah, F. Khellaf, J. M. Létang, S. Rit, I. Rinaldi, A comprehensive theoretical comparison of proton imaging set-ups in terms of spatial resolution, Physics in Medicine \& Biology 63 (2018) 135013. URL: http: //iopscience.iop.org/article/10.1088/1361-6560/aaca1fhttp: //stacks.iop.org/0031-9155/63/i=13/a=135013?key=crossref . 8cd18401666e345b9e2df906530658cd. doi:10.1088/1361-6560/aaca1f.

[80] U. Schneider, P. Pemler, J. Besserer, E. Pedroni, A. Lomax, B. KaserHotz, Patient specific optimization of the relation between CT-Hounsfield units and proton stopping power with proton radiography, Medical Physics 32 (2004) 195-199. URL: http://doi.wiley.com/10.1118/1. 1833041, doi $10.1118 / 1.1833041$.

${ }_{1150}$ [81] P. J. Doolan, M. Testa, G. Sharp, E. H. Bentefour, G. Royle, H. M. Lu, Patient-specific stopping power calibration for proton therapy planning based on single-detector proton radiography, Physics in Medicine and Biology (2015). doi 10.1088/0031-9155/60/5/1901 
[82] H. M. Lu, A point dose method for in vivo range verification in pro1155 ton therapy, Physics in Medicine and Biology (2008). doi 10.1088/ 0031-9155/53/23/N01

[83] B. Gottschalk, S. Tang, E. H. Bentefour, E. W. Cascio, D. Prieels, H. M. $\mathrm{Lu}$, Water equivalent path length measurement in proton radiotherapy using time resolved diode dosimetry, Medical Physics (2011). doi:10. $1118 / 1.3567498$.

[84] M. Testa, J. M. Verburg, M. Rose, C. H. Min, S. Tang, E. H. Bentefour, H. Paganetti, H.-M. Lu, Proton radiography and proton computed tomography based on time-resolved dose measurements, Physics in Medicine and Biology 58 (2013) 8215-8233. URL: https: //iopscience.iop.org/article/10.1088/0031-9155/58/22/8215. doi $10.1088 / 0031-9155 / 58 / 22 / 8215$.

[85] R. Zhang, G. C. Sharp, K. W. Jee, E. Cascio, J. Harms, J. B. Flanz, H. M. Lu, Iterative optimization of relative stopping power by single detector based multi-projection proton radiography, Physics in Medicine and Biology 64 (2019). doi 10.1088/1361-6560/aaf976.

[86] C. A. Collins-Fekete, S. Brousmiche, D. C. Hansen, L. Beaulieu, J. Seco, Pre-treatment patient-specific stopping power by combining list-mode proton radiography and x-ray CT, Physics in Medicine and Biology 62 (2017) 6836-6852. doi 10.1088/1361-6560/aa7c42.

1175 [87] N. Krah, V. Patera, S. Rit, A. Schiavi, I. Rinaldi, Regularised patientspecific stopping power calibration for proton therapy planning based on proton radiographic images, Physics in Medicine and Biology 64 (2019). doi $10.1088 / 1361-6560 / a b 03 d b$.

[88] N. Krah, S. Rit, Optimized conversion from CT numbers to proton relative stopping power based on proton radiography and scatter corrected cone-beam CT images, 15th International Meeting on Fully Three-Dimensional Image Reconstruction in Radiology and Nuclear Medicine (2019) 96. URL: https://www.spiedigitallibrary. org/conference-proceedings-of-spie/11072/2534898/

Optimized-conversion-from-CT-numbers-to-proton-relative-stopping-power/ 10.1117/12.2534898.full, doi $10.1117 / 12.2534898$,

[89] P. Palaniappan, S. Meyer, F. Kamp, C. Belka, M. Riboldi, K. Parodi, C. Gianoli, Deformable image registration of the treatment planning CT with proton radiographies in perspective of adaptive proton

1190 therapy, Physics in Medicine \& Biology Epub (2020). URL: https:

1. //iopscience.iop.org/article/10.1088/1361-6560/ab8fc3. doi:10. 1088/1361-6560/ab8fc3.

[90] H. Nikjoo, S. Uehara, W. E. Wilson, M. Hoshi, D. T. Goodhead, Track structure in radiation biology: Theory and applications, International 
Journal of Radiation Biology 73 (1998) 355-364. URL: https://doi .org/ 10.1080/095530098142176 doi $10.1080 / 095530098142176$.

[91] A. Mairani, S. Brons, F. Cerutti, A. Fassò, A. Ferrari, M. Krämer, K. Parodi, M. Scholz, F. Sommerer, The FLUKA Monte Carlo code coupled with the local effect model for biological calculations in carbon ion therapy, Physics in Medicine \& Biology 55 (2010) 4273-4289. URL: https: //iopscience.iop.org/article/10.1088/0031-9155/55/15/006/.

[92] 1990 Recommendations of the International Commission on Radiological Protection., Annals of the ICRP (1991). doi:10.1016/0720-048x(92) 90214-t.

[93] D. J. Carlson, R. D. Stewart, V. A. Semenenko, G. A. Sandison, Combined Use of Monte Carlo DNA Damage Simulations and Deterministic Repair Models to Examine Putative Mechanisms of Cell Killing, Radiation Research 169 (2008) 447-459. URL: https://doi.org/10.1667/RR1046.1.

[94] T. T. Böhlen, F. Cerutti, M. P. W. Chin, A. Fassò, A. Ferrari, P. G. Ortega, A. Mairani, P. R. Sala, G. Smirnov, V. Vlachoudis, The FLUKA Code: Developments and Challenges for High Energy and Medical Applications, Nuclear Data Sheets 120 (2014) 211214. URL: https://www.sciencedirect.com/science/article/pii/ S0090375214005018?via\%3Dihub.

[95] L. Yasui, N. Luckett, M. Pankuch, J. Edwards, J. Ramos-Mendez, R. Johnson, B. Faddegon, E. Blakely, R. Schulte, DNA Double-Strand Break Induction and Repair of Proton Computed Tomography in Normal Human Cells, in: Radiological Society of North America 2019 Scientific Assembly and Annual Meeting, 2019. URL: archive.rsna.org/2019/ 19009809.html.

[96] L. Johnson, B. Keeney, G. Ross, H.-W. Sadrozinski, A. Seiden, D. Williams, L. Zhang, V. Bashkirov, R. Schulte, K. Shahnazi, Initial studies on proton computed tomography using a silicon strip detector telescope, Nuclear Instruments and Methods in Physics Research Section A: Accelerators, Spectrometers, Detectors and Associated Equipment 514 (2003) 215-223. URL: https://linkinghub.elsevier.com/retrieve/ pii/S0168900203023970 doi:10.1016/j.nima.2003.08.108

[97] G. Cirrone, G. Candiano, G. Cuttone, S. Lo Nigro, D. Lo Presti, N. Randazzo, V. Sipala, M. Russo, S. Aiello, M. Bruzzi, D. Menichelli, 1230 M. Scaringella, S. Miglio, M. Bucciolini, C. Talamonti, S. Pallotta, The Italian project for a proton imaging device, $\mathrm{Nu}-$ clear Instruments and Methods in Physics Research Section A: Accelerators, Spectrometers, Detectors and Associated Equipment 576 (2007) 194-197. URL: https://linkinghub.elsevier.com/retrieve/ pii/S0168900207002458, doi:10.1016/j.nima.2007.01.151. 
[98] T. Price, M. Esposito, G. Poludniowski, J. Taylor, C. Waltham, D. Parker, S. Green, S. Manolopoulos, N. Allinson, T. Anaxagoras, P. Evans, J. Nieto-Camero, Expected proton signal sizes in the PRaVDA Range Telescope for proton Computed Tomography, $\mathrm{P} 05013$. $a=$ 05013?key=crossref .f86c76fb3dc82f 4 e8c0f681f $3972 a 914$ doi $10.1088 / 1748-0221 / 10 / 05 /$ P05013.

[99] T. Price, PRaVDA: High Energy Physics towards proton Computed Tomography, Nuclear Instruments and Methods in Physics Research Section A: Accelerators, Spectrometers, Detectors and Associated Equipment 824 (2016) 226-227.

URL: http://dx.doi.org/10.1016/j.nima.2015.12.013https: //linkinghub.elsevier.com/retrieve/pii/S0168900215015843. doi $10.1016 / j$.nima.2015.12.013

[100] M. Esposito, T. Price, T. Anaxagoras, N. Allinson, Geant4based simulations of charge collection in CMOS Active Pixel Sensors, Journal of Instrumentation 12 (2017) P03028-

1 P03028. URL: http://stacks.iop.org/1748-0221/12/i=03/ $\mathrm{a}=\mathrm{P} 03028$ ?key=crossref .98cece91d4f57e220f 48171d89191862 doi $10.1088 / 1748-0221 / 12 / 03 /$ P03028.

[101] M. Esposito, T. Price, J. T. Taylor, C. Waltham, S. Manger, B. Phoenix, G. Poludniowski, S. Green, P. M. Evans, P. P. Allport, S. Manolopoulos, J. Nieto-Camero, N. M. Allinson, Abstract ID: 139 Monte Carlo simulations for imaging in proton therapy, Physica Medica 42 (2017) 30. URL: https://linkinghub.elsevier.com/retrieve/pii/ S1120179717303940 doi:10.1016/j.ejmp.2017.09.075

[102] H. Pettersen, J. Alme, A. Biegun, A. van den Brink, M. Chaar, D. Fehlker, I. Meric, O. Odland, T. Peitzmann, E. Rocco, K. Ulla1265 land, H. Wang, S. Yang, C. Zhang, D. Röhrich, Proton tracking in a high-granularity Digital Tracking Calorimeter for proton CT purposes, Nuclear Instruments and Methods in Physics Research Section A: Accelerators, Spectrometers, Detectors and Associated Equipment

1. 860 (2017) 51-61. URL: https://linkinghub.elsevier.com/retrieve/ 1270 pii/S0168900217301882, doi:10.1016/j.nima.2017.02.007

[103] H. F. Sadrozinski, R. P. Johnson, S. MacAfee, A. Plumb, D. Steinberg, A. Zatserklyaniy, V. A. Bashkirov, R. F. Hurley, R. W. Schulte, Development of a head scanner for proton CT, in: Nuclear Instruments and Methods in Physics Research, Section A: Accelerators, Spectrometers, Detectors and Associated Equipment, 2013. doi 10.1016/j.nima.2012.04. 029 . 
[104] R. P. Johnson, J. Dewitt, C. Holcomb, S. Macafee, H. F. Sadrozinski, D. Steinberg, Tracker readout ASIC for proton computed tomography

n data acquisition, IEEE Transactions on Nuclear Science (2013). doi 10 . 1109/TNS.2013.2274663.

[105] V. Giacometti, V. A. Bashkirov, P. Piersimoni, S. Guatelli, T. E. Plautz, H. F. Sadrozinski, R. P. Johnson, A. Zatserklyaniy, T. Tessonnier, K. Parodi, A. B. Rosenfeld, R. W. Schulte, Software platform for simulation of a prototype proton CT scanner, Medical Physics 44 (2017) 1002-1016. URL: http://doi.wiley.com/10.1002/mp.12107. doi:10.1002/mp.12107.

[106] J. B. Birks, Scintillations from Organic Crystals: Specific Fluorescence and Relative Response to Different Radiations, Proceedings of the Physical Society. Section A 64 (1951) 874-877. URL: http://stacks.iop.org/0370-1298/64/i=10/a=303?key=crossref .

1290 f6127795b0efda6211aab9d5c1b9ae31 doi 10.1088/0370-1298/64/10/ 303.

[107] V. Giacometti, S. Guatelli, M. Bazalova-Carter, A. Rosenfeld, R. Schulte, Development of a high resolution voxelised head phantom for medical physics applications, Physica Medica 33 (2017) 182-188.

1295 URL: http://dx.doi.org/10.1016/j.ejmp.2017.01.007https: //linkinghub.elsevier.com/retrieve/pii/S1120179717300078. doi $10.1016 /$ j.ejmp. 2017.01.007.

[108] S. Meyer, C. Gianoli, L. Magallanes, B. Kopp, T. Tessonnier, G. Landry, G. Dedes, B. Voss, K. Parodi, Comparative Monte Carlo study on the

${ }_{1300}$ performance of integration- and list-mode detector configurations for carbon ion computed tomography, Physics in Medicine and Biology

(1) 62 (2017) 1096-1112. URL: http://stacks.iop.org/0031-9155/62/ $i=3 / a=1096$ ?key=crossref.c14e328466ec50a0e74a2649307d033d. doi $10.1088 / 1361-6560 /$ aa5602.

1305 [109] G. Dedes, L. De Angelis, S. Rit, D. Hansen, C. Belka, V. Bashkirov, R. P. Johnson, G. Coutrakon, K. E. Schubert, R. W. Schulte, K. Parodi, G. Landry, Application of fluence field modulation to proton computed tomography for proton therapy imaging, Physics in Medicine \& Biology 62 (2017) 6026-6043. URL:https://doi.org/10.1088/1361-6560/aa7734. doi $10.1088 / 1361-6560 /$ aa7734.

[110] P. Piersimoni, J. Ramos-Méndez, T. Geoghegan, V. A. Bashkirov, R. W. Schulte, B. A. Faddegon, The effect of beam purity and scanner complexity on proton CT accuracy, Medical Physics 44 (2017) 284-298. URL: http://doi.wiley.com/10.1002/mp.12013, doi:10.1002/mp.12013.

[111] T. E. Plautz, V. Bashkirov, V. Giacometti, R. F. Hurley, R. P. Johnson, P. Piersimoni, H. F. Sadrozinski, R. W. Schulte, A. Zatserklyaniy, An evaluation of spatial resolution of a prototype proton CT scanner, Medical 
Physics 43 (2016) 6291-6300. URL: http://doi.wiley.com/10.1118/1. 4966028, doi $10.1118 / 1.4966028$.

${ }_{1320}$ [112] S. N. Penfold, A. B. Rosenfeld, R. W. Schulte, H. F. Sadrozinksi, Geometrical optimization of a particle tracking system for proton computed tomography, Radiation Measurements 46 (2011) 2069-2072. URL: http://dx.doi.org/10.1016/j.radmeas.2011.04.032 doi 10 . 1016/j.radmeas.2011.04.032.

1325

1330

1335

[114] J. Dickmann, S. Rit, M. Pankuch, R. P. Johnson, R. W. Schulte, K. Par-

1906. URL: https://onlinelibrary.wiley.com/doi/abs/10.1002/mp.

[113] H.-W. Sadrozinski, T. Geoghegan, E. Harvey, R. Johnson, T. Plautz, A. Zatserklyaniy, V. Bashkirov, R. Hurley, P. Piersimoni, R. Schulte, P. Karbasi, K. Schubert, B. Schultze, V. Giacometti, Operation of the preclinical head scanner for proton CT, Nuclear Instruments and Methods in Physics Research Section A: Accelerators, Spectrometers, Detectors and Associated Equipment 831 (2016) 394-399. URL: http://dx.doi.org/10.1016/j.nima.2016.02.001https: //linkinghub.elsevier.com/retrieve/pii/S0168900216001455. doi $10.1016 / j$.nima.2016.02.001. odi, G. Dedes, G. Landry, An optimization algorithm for dose reduction with fluence-modulated proton CT, Medical Physics 47 (2020) 189514084 doi $10.1002 / \mathrm{mp} .14084$.

[115] L. Volz, C. A. Collins-Fekete, P. Piersimoni, R. P. Johnson, V. Bashkirov, R. Schulte, J. Seco, Stopping power accuracy and achievable spatial resolution of helium ion imaging using a prototype particle CT detector system, Current Directions in Biomedical Engineering 3 (2017) 401-404. doi 10.1515/cdbme-2017-0084. 